\title{
The Estrogen-Induced miR-19 Downregulates Secretory Leucoprotease Inhibitor Expression in Monocytes
}

\author{
Paul J. McKiernan ${ }^{a}$ Stephen G.J. Smith ${ }^{b}$ Andrew L. Durham ${ }^{c}$ Ian M. Adcock ${ }^{c}$ \\ Noel G. McElvaney ${ }^{a}$ Catherine M. Greene ${ }^{d}$ \\ ${ }^{a}$ Department of Medicine, Royal College of Surgeons in Ireland, Beaumont Hospital, Dublin, Ireland; ${ }^{b}$ Department of \\ Clinical Microbiology, School of Medicine, Trinity College Dublin, Dublin, Ireland; ' Airways Disease Section, \\ National Heart and Lung Institute, Imperial College London, London, UK; ${ }^{d}$ Department of Clinical Microbiology, \\ Royal College of Surgeons in Ireland, Beaumont Hospital, Dublin, Ireland
}

\author{
Keywords \\ Estrogen - microRNA - Secretory leucoprotease inhibitor . \\ Monocytes
}

\begin{abstract}
Compared to females, males are more susceptible to acute viral and other respiratory tract infections that display greater severity and higher mortality. In contrast, females tend to fare worse with chronic inflammatory diseases. Circulating $17 \beta$-estradiol (E2) is a female-specific factor that may influence the progression of human lung diseases. Here we hypothesize that E2 modulates the inflammatory response of monocytes through microRNA (miRNA)-based modulation of secretory leucoprotease inhibitor (SLPI), an antiprotease with immunomodulatory effects. Monocytic cells were treated $\pm E 2$, and differentially expressed miRNAs were identified using PCR profiling. Cells were transfected with miRNA mimics or antimiRs and SLPI mRNA and protein levels were quantified. Luciferase activity assay using wildtype and $\Delta$ miR$19 \mathrm{a} / \mathrm{b}-\mathrm{SLPI3}$ 'UTR reporter constructs and chromatin immunoprecipitation on E2-treated monocytes were performed. E2 downregulated SLPI and upregulated miR-19 expression in monocytes. Transfection with premiR-19b reduced SLPI
\end{abstract}

mRNA and protein levels and this effect was abrogated using antimiRs against miR-19b. miR-19b directly binds the SLPI $3^{\prime}$ UTR. The mechanism responsible for E2-mediated upregulation of miR-19 occurs via increased MIR17HG promoter activity mediated by c-MYC. Overall E2 decreases SLPI expression in human monocytic cells, via changes in miRNA expression and highlights the potential for estrogen to modulate the innate immune system.

(C) 2019 The Author(s)

Published by S. Karger AG, Basel

\section{Introduction}

All species have sex-related differences in physical characteristics and biological activities [1]. Females on average live longer than men, and as people age, the proportions of women at a particular age increases compared to men [2]. It is now well recognised that females have a lower burden of viral, bacterial and parasitic infections, particularly during reproductive years, and yet experience a higher prevalence of autoimmune diseases [3]. With focus on respiratory illnesses, males outnumber females in the majority of acute inflammatory diseases and have poorer prognoses [4]. In contrast, females fare worse 
with chronic inflammatory diseases, and it has been hypothesised that a higher inflammatory response in females is protective where acute conditions are concerned, but chronic inflammation may cause deleterious tissue damage [5]. Male versus female dichotomy is well recognised in chronic inflammatory lung diseases such as cystic fibrosis (CF), chronic obstructive pulmonary disease (COPD) and asthma. Female sex has been recognised as a negative prognostic factor in CF [6, 7]. In 1996, Corey and Farewell analysed almost $4000 \mathrm{CF}$ patients from the Canadian registry between 1970 and 1989 and detected a diminished survival of females of $>5$ years compared to males over this period [8]. Poorer survival for females with CF was also confirmed in a UK study [9], and despite significant improvements in CF survival over the last few decades, females have continued to display a lower median survival age compared to males $[6,10-12]$. Females with CF also have poorer lung function, higher mortality, earlier colonisation with Pseudomonas aeruginosa, deteriorate with Burkholderia earlier than males [11, 13-15], require more intensified antibiotic treatments, greater hospitalisation rates and have a higher risk of $P$. aeruginosa and Burkholderia colonisation of the lung than that of males [15-17]. Furthermore, females with CF exhibit earlier $P$. aeruginosa mucoid conversion than that of males, leading to worse clinical outcomes [17]. However, some studies report no male versus female differences in children $[18,19]$. Therefore, recently, the focus has turned to the role of sex steroid hormones to potentially explain the CF gender gap. Our group has shown that estrogen induces the conversion of $P$. aeruginosa to mucoidy in vitro and is associated with increased exacerbations and mucoid conversion in vivo [20].

Sex differences also exist in other chronic inflammatory lung diseases. The prevalence of asthma is lower in females than in males before adolescence; however, this trend is reversed post-puberty [21-23]. In the United States and elsewhere, $>60 \%$ of all adult patients with asthma are women, and females asthmatics are more likely to experience hospitalisations and more likely to die from asthma [24]. A similar gender gap is emerging in COPD possibly due in part to the increase in female smokers. Females have now surpassed males with respect to COPD mortality [25]. In oxygen-dependent patients with severe COPD, women have a $50 \%$ higher risk of mortality compared with men [26]. Sex hormones, including estrogen, may contribute to the progression of these inflammatory lung diseases in women.

Of the 3 major forms circulatory forms of estrogen in females, $17 \beta$-estradiol (E2) is the most potent and is the

Estrogen-Induced miR-19b Regulates

SLPI in Monocytes predominant estrogen in non-pregnant pre-menopausal females. Our group has demonstrated that E2 induces Toll-like receptor hypo-responsiveness in CF bronchial epithelial cells to a range of bacterial agonists [5]. This manifests as an inhibition of interleukin- 8 release and was found to be the result of an estrogen receptor (ER) $\beta$-mediated upregulation of the expression of secretory leucoprotease inhibitor (SLPI), an important anti-protease widely expressed in the lung, mucosal secretions and the skin. SLPI has been found to be a multifunctional protein; it is a serine protease inhibitor that can protect tissue from degradation by a number of proteases such as neutrophil elastase, cathepsin G and trypsin, and studies in myeloid cells have demonstrated its ability to block NF$\kappa \mathrm{B}$ activation and pro-inflammatory signalling [27-29]. Estrogen has been shown to increase the expression of SLPI in other cell types, including uterine epithelial cells $[30,31]$. SLPI, however, is also expressed in non-epithelial cells such as monocytes. What remains unknown is whether estrogen regulates SLPI expression in these key immune cells.

SLPI expression is potentially regulated by microRNAs (miRNA). These are small RNAs involved in the regulation of gene expression at the translational level. A single gene may be targeted by multiple miRNAs and one miRNA targets many (typically over 100) mRNAs. Given that $30-80 \%$ of human genes are predicted to be influenced by miRNA, it is likely that miRNAs regulate SLPI expression. SLPI mRNA appears not to be alternatively spliced, with no known transcript variants or alternative $3^{\prime}$ UTRs, lending itself as an ideal target for miRNA regulation. Estrogen has been shown to regulate the expression of a wide variety of miRNAs, in a variety of cell types [32-36] and it is unknown yet whether any estrogen-regulated miRNAs target SLPI.

SLPI is especially present at mucosal surfaces. Thus, it is important to elucidate the mechanisms that regulate macrophage-derived SLPI expression in specific contexts, particularly in the lung. As a starting point to address whether E2 affects SLPI expression in monocytes - the precursors of tissue and lung macrophages we explored the effect of E2 on SLPI in monocytic cell lines. Here we also describe the effects of E2 on SLPI expression in primary human monocytes. We profile global miRNA expression in THP-1 cells in response to E2. To this end, 2 of the upregulated miRNAs are explored further in a second monocytic cell line and in primary monocytes; miR-19a and miR-19b, which are encoded as part of an important 6-miRNA miR-17-92 cluster on chromosome 13 [37]. Predicted to target the $3^{\prime} \mathrm{UTR}$ of human 
Table 1. Primer pairs used in this study

\begin{tabular}{|c|c|c|}
\hline mRNA/gDNA & Primers $\left(5^{\prime}-3^{\prime}\right)$ & $\begin{array}{l}\text { Annealing temperature } \\
\left({ }^{\circ} \mathrm{C}\right)\end{array}$ \\
\hline GAPDH & $\begin{array}{l}\text { (F)-CATGAGAAGTATGACAACAGCCT } \\
\text { (R)-AGTCCTTCCACGATACCAAAGT }\end{array}$ & 57 \\
\hline SLPI & $\begin{array}{l}\text { (F)-AATGCCTGGATCCTGTTGAC } \\
\text { (R)-AAAGGACCTGGACCACACAG }\end{array}$ & 57 \\
\hline MIR17HG & $\begin{array}{l}\text { (F)-GCCCAATCAAACTGTCCTGT } \\
\text { (R)-ACCGATCCCAACCTGTGTAG }\end{array}$ & 57 \\
\hline MIR17HG Ebox1 (ChIP) & $\begin{array}{l}\text { (F)-AAAGGCAGGCTCGTCGTTG } \\
\text { (R)-CGGGATAAAGAGTTGTTTCTCCAA }\end{array}$ & 55 \\
\hline
\end{tabular}

SLPI, secretory leucoprotease inhibitor; ChIP, chromatin immunoprecipitation.

SLPI, the effect of modulation of the levels of miR-19a and miR-19b on SLPI expression is described and their ability to bind directly to the $3^{\prime} \mathrm{UTR}$ of SLPI mRNA is also evaluated. Finally, the mechanism of E2-induced upregulation of the miR-17-92 cluster is investigated.

\section{Methods}

\section{Cell Culture and Treatments}

All cell lines were maintained at $37^{\circ} \mathrm{C}$ in a humidified $\mathrm{CO}_{2}$ incubator in appropriate media. THP-1 (human acute monocytic leukaemia cell line), U937 (Human monocytic [histiocytic lymphoma] cell line) and HEK293 (human embryonic kidney cell line) cells were obtained from the European Collection of Cell Cultures (Salisbury, UK). THP-1 and U937 cells were cultured in RPMI 1640 and HEK293 cells were cultured in Dulbecco's modified Eagle's medium (Sigma-Aldrich, St. Louis, MO, USA). All culture media contained 10\% foetal calf serum (Gibco, Billings, MT) and $1 \%$ penicillin/streptomycin (Invitrogen, Carlsbad, CA, USA). Before treatment, cells were serum starved for at least $2 \mathrm{~h}$. Cell culture grade $17 \beta$-E2 (Sigma-Aldrich) was added cells at a final concentration of $10^{-8} \mathrm{M}$. Equivalent ethanol controls were $0.00028 \%$.

\section{Isolation and Treatment of Primary Human Monocytes}

Following informed consent under a protocol approved by Beaumont Hospital Ethics Committee peripheral blood monocytes were isolated from heparinised venous peripheral blood obtained from 6 healthy male individuals. About $15 \mathrm{~mL}$ of blood was mixed with an equal volume of $0.9 \% \mathrm{NaCl}$ and the diluted blood was carefully layered over Lymphoprep (Axis Shield, Dundee, Scotland) in a fresh $50 \mathrm{~mL}$ tube, avoiding mixing of blood and Lymphoprep before centrifugation. Ficoll-Paque Plus density gradient centrifugation was carried out at $800 \times g$ for $10 \mathrm{~min}$ at room temperature with the brake off. The mononuclear cell band was aspirated, washed in Hank's Balanced Salt Solution (Lonza, Basel, Switzerland) and centrifuged at $500 \times g$ for $5 \mathrm{~min}$. These mononuclear cells were then resuspended in $1 \mathrm{~mL}$ recommended medium (1 mM EDTA, 2\% foetal calf serum in Dulbecco's phosphate- buffered saline). Monocytes were purified from this mononuclear cell population using the EasySep ${ }^{\circledR}$ Human CD14 Selection Cocktail (StemCell Technologies, Grenoble, France) as per the manufacturer's protocol.

\section{miRNA Expression Profiling}

Total RNA was isolated using TRI reagent (Sigma-Aldrich) according to the manufacturer's instructions. miRNA profiling was carried out by TaqMan Low Density arrays (TLDA). Prior to miRNA expression profiling, RNA from triplicates treatments was pooled, and miRNA expression profiling was performed by a stem-loop real-time PCR-based miRNA expression profiling method using the TaqMan miRNA Arrays version 3.0 (4444913) from Applied Biosystems (Austin, TX, USA). Two array cards (A and $B$ ) for each sample were run on the Applied Biosystems 7900HT fast real-time PCR system, which measured expression levels of 754 different human miRNA in each sample and 4 endogenous controls. RNA (20 ng) from pooled samples was reverse transcribed with the Megaplex ${ }^{\mathrm{TM}}$ primer pool allowing simultaneous reverse transcription of 754 miRNA and 4 endogenous controls across 2 RT pools on a Thermal cycler. A pre-amplification step was performed on the Megaplex ${ }^{\mathrm{TM}} \mathrm{RT}$ product $(5 \mu \mathrm{L})$ using TaqMan ${ }^{\circledR}$ PreAmp Master Mix $(2 \times)$ and PreAmp Primer Mix $(10 \times)$ in a $25 \mu \mathrm{L}$ reaction on the $7900 \mathrm{HT}$ fast real-time PCR system The PreAmp primer pool contained forward primers specific for each miRNA and a universal reverse primer. Specific Megaplex ${ }^{\mathrm{TM}}$ primer pools and PreAmp Primer Mix were used for A and B cards. PCR for TLDA was carried out with a sample specific cDNA pool, made up to $100 \mu \mathrm{L}\left(25 \mu \mathrm{L}\right.$ of PreAmp reaction and $75 \mu \mathrm{L}$ of $\left.\mathrm{H}_{2} \mathrm{O}\right)$ and combined with TaqMan ${ }^{\circledR} 2 \times$ Universal PCR Master Mix. One hundred microliter of each sample was added to the 8 fill ports on each card to populate individual wells. The cards were sealed, centrifuged and then run on the $7900 \mathrm{HT}$.

\section{Quantitative Reverse Transcription-PCR for $m R N A$ and} miRNA Levels

For quantification of mRNA, equal quantities of RNA were reverse transcribed into cDNA using the Quantitect Reverse Transcription kit (Qiagen, Valencia, CA, USA). The resulting cDNA was used as template for quantitative real-time PCR. Oligonucleotide primers were synthesized (MWG Biotech, Ebersberg, Ger-
92

Innate Immun 2020;12:90-102

DOI: $10.1159 / 000500419$
McKiernan/Smith/Durham/Adcock/ McElvaney/Greene 
many) and quantitative PCR reactions performed in $20 \mu \mathrm{L}$ containing $2 \mu \mathrm{L}$ of template cDNA, SYBR Green MasterMix (Roche, Basel, Switzerland) and $10 \mathrm{pmol}$ of each primer (Table 1).

Individual miRNA levels were measured using Taqman miRNA assays (Applied Biosystems, Austin, TX, USA) according to the manufacturer's instructions. Amplification for both mRNA and miRNA was performed on the Roche LC480 Lightcycler in triplicate samples, including no-template controls. Relative expression of transcripts and miRNAs relative to GAPDH and U6 snRNA, respectively, were determined using the $2^{-\Delta \Delta C t}$ method,

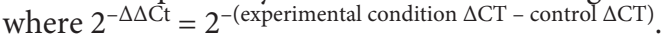

Transfection of Pre-miRs, anti-miRs and Reporter Plasmids

U937 cells $\left(1 \times 10^{5}\right.$ in triplicate $)$ were left non-transfected or transiently transfected $(48 \mathrm{~h})$ with indicated concentrations of a negative control pre-miR, synthetic pre-miRs or anti-miRs (Ambion, Life Technologies) using Ribojuice (Novagen, Madison, WI, USA) in OptiMEM-reduced serum media (Life Technologies, Carlsbad, CA, USA). RNA was isolated for quantitative reverse transcription-PCR (qRT-PCR) and Taqman miRNA assays, whereas supernatants were recovered for ELISA.

The SLPI 3'UTR was cloned immediately downstream of the coding sequence of Firefly luciferase in the pMIR-REPORT (Origene, Rockville, MD, USA) reporter plasmid, to create pMIRSLPI-3'UTR. A mutant of this construct lacking the miR-19a/b-3p binding site in the SLPI $3^{\prime}$ UTR was generated by inverse PCR using primers flanking the $7 \mathrm{bp}$ predicted miRNA recognition element (MRE) for miR-19. This approach utilises a pair of back-to-back 5 'phosphorylated primers, facing outward, each binding to one of the DNA strands of a circular plasmid. The entire plasmid is PCR amplified, using these primers. For this study, these primers were designed to create a 7 base pair deletion in the miR-19a/b binding site in the SLPI $3^{\prime} U T R$ (base 170-177), and the following primers were used which flank each side of this sequence: SLPI-MUT-F 5'-AAAGCTTAATAAAGGATCTTTTATTTTCATTGG- ${ }^{\prime}$ ' and SLPI-MUT-R 5'-GAGAAATAGGCTCGTTTATTTATTC- ${ }^{\prime}$. The absence of the miRNA binding site in this mutant construct, pMIR-SLPI-3'UTR- $\Delta 19 a / b$, was verified by DNA sequencing. HEK293 cells $\left(1 \times 10^{5}\right.$ in triplicate) were transiently transfected with $250 \mathrm{ng}$ pMIR-SLPI-3'UTR or pMIR-SLPI-3'UTR- $\Delta 19 a / b$ and $100 \mathrm{ng}$ of the reference Renilla luciferase reporter plasmid pRLSV40 (Promega, Madison, WI, USA) with Genejuice (Novagen). Lysates were prepared and assayed for both Firefly and Renilla luciferase using the Luciferase assay system (Promega) and coelenterazine (Marker Gene Technologies, Eugene, OR, USA). Firefly luciferase activity was normalized to the Renilla luciferase activity.

\section{SLPI ELISA}

SLPI protein concentrations in cell supernatants were determined by sandwich ELISA using specific antibodies to SLPI (R\&D Systems, Minneapolis, MN, USA).

\section{Chromatin Immunoprecipitation Analysis}

Chromatin immunoprecipitation (ChIP) analysis was carried using the High Cell Number protein A ChIP kit following the manufacturer's instructions (Diagenode, Ougrée, Belgium). In brief, cells were cultured and treated as described above. Following stimulation the cells were formalin-fixed. The cells were sonicated to shear DNA $(12 \times 15 \mathrm{~s}$ at $40 \%$ amplitude $)$ to approximately $200-$

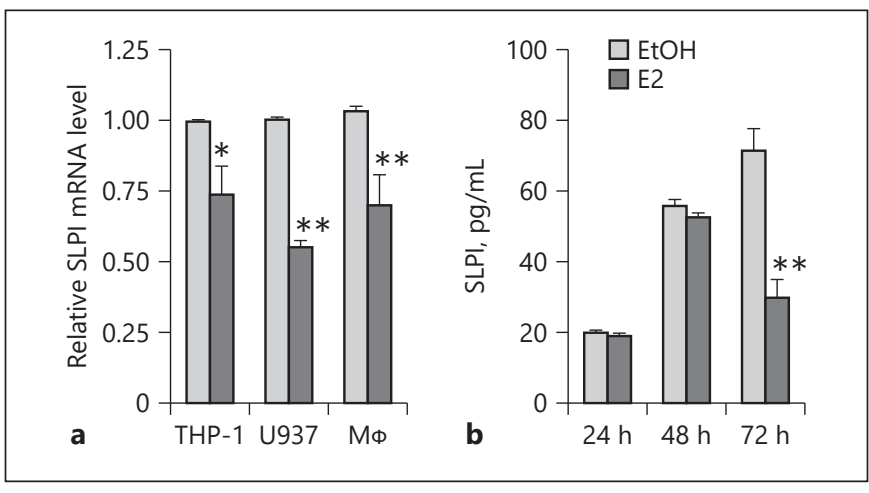

Fig. 1. SLPI expression in monocytes in response to estrogen treatment. Cells were treated, in triplicate, with E2 $\left(10^{-8} \mathrm{M}\right)$ or with EtOH. a Relative levels of SLPI expression in THP-1 $(n=4)$ and U937 $(n=3)$ monocytic cell lines and peripheral blood monocytes $(n=6)$ in response to $\mathrm{E} 2$ treatment after $6 \mathrm{~h}$, as determined by qRTPCR. Results (mean \pm SEM) are normalised to GAPDH expression. b SLPI protein levels in U937 monocytic cell supernatants in response to E2 treatment, as determined by ELISA. ${ }^{*} p \leq 0.05$, ${ }^{* *} p \leq 0.01$; one-tailed $t$ test. SLPI, secretory leucoprotease inhibitor; EtOH, ethanol vehicle control; E2, estradiol.

1,000 bp in length. ER $\alpha$ and c-MYC were immunoprecipitated using $1 \mu \mathrm{g}$ of antibody (Santa Cruz Biotechnology, Santa Cruz, CA, USA). After reversal of cross-linking, the DNA was purified and quantitative PCR was carried out as described above. The amount of DNA recovered by ChIP was normalized to an input control. IgG isotype antibodies (provided by Diagenode) were used as a negative control for the ChIP assay.

\section{Statistical Analysis}

All analyses were performed using GraphPad Prism 4.0 software package (San Diego, CA, USA). Results are expressed as the mean \pm SEM and were compared by Student $t$ test (non-parametric, 1 tailed) or 2-way ANOVA, as appropriate. Differences were considered significant at $p$ values of $\leq 0.05$.

\section{Results}

\section{Estrogen Downregulates SLPI in Monocytic Cell Lines} and in Primary Monocytes

$17 \beta-E 2$ has previously been shown to increase the expression of SLPI in bronchial epithelial cells [5]. To examine the effect of E2 on the expression of SLPI in monocytes, 2 monocytic cell lines were treated with either $10^{-8}$ M E2 or ethanol vehicle control (EtOH). E2 significantly downregulated SLPI mRNA abundance in both cell lines examined (THP- $1 ; p<0.05 ; n=4$ and U937; $p<0.01 ; n=$ 3), as determined by qRT-PCR (Fig. 1a). E2 also significantly downregulated the expression of SLPI mRNA in primary monocytes obtained from the peripheral blood 
Table 2. miRNAs downregulated in THP-1 monocytic cells in response to estrogen treatment

\begin{tabular}{ll}
\hline miRNA & Fold change (down) \\
\hline hsa-miR-519b-3p & $-5.81 \mathrm{E}+05$ \\
hsa-miR-135a & $-2.37 \mathrm{E}+04$ \\
hsa-miR-29c & -10.04 \\
hsa-miR-523 & -9.39 \\
hsa-miR-378 & -7.57 \\
hsa-miR-203 & -7.41 \\
hsa-miR-646 & -7.06 \\
hsa-miR-454 & -4.38 \\
hsa-miR-205 & -4.06 \\
hsa-miR-377 & -3.98 \\
hsa-miR-324-3p & -3.96 \\
hsa-miR-628-3p & -3.23 \\
hsa-miR-544 & -3.10 \\
hsa-let-7e & -3.06 \\
hsa-miR-1208 & -2.86 \\
hsa-miR-302b & -2.76 \\
hsa-miR-335* & -2.53 \\
hsa-miR-571 & -2.52 \\
hsa-miR-639 & -2.48 \\
hsa-miR-1260 & -2.02 \\
hsa-miR-200a & -1.85 \\
hsa-miR-628-5p & -1.80 \\
hsa-let-7d & -1.78 \\
hsa-miR-575 & -1.76 \\
hsa-miR-320B & -1.68 \\
\hline
\end{tabular}

RNA from triplicate $\mathrm{E} 2\left(10^{-8} \mathrm{M}\right)$ or EtOH $6 \mathrm{~h}$ treatments carried out in THP-1 cells was pooled and profiled by TaqMan ${ }^{\circledR}$ Low Density Array. Relative quantification was determined using the $2^{-\Delta \Delta \mathrm{Ct}}$ method.

miRNA, microRNA. miR* (i.e., miR-star) is the old nomenclature for a passenger, rather than a guide strand.

$(p<0.01 ; n=6 ; p<0.01 ; n=6)$. SLPI protein expression from U937 cells increased over time as determined by ELISA (Fig. 1b) A similar effect was observed in THP-1 cells where SLPI protein levels increased from $90 \pm 12 \mathrm{pg} /$ $\mathrm{mL}$ at $6 \mathrm{~h}$ to $180 \pm 5 \mathrm{pg} / \mathrm{mL}$ at $24 \mathrm{~h}$ (data not shown). E2 significantly decreased SLPI protein secretion in U937 monocytes at later time points ( $\mathrm{T}=72 \mathrm{~h}, p<0.01$; Fig. $1 \mathrm{~b}$ ).

\section{Putative SLPI-Targeting miRNAs miR-19a and}

miR-19b Are Increased in Monocytic Cells in Response to E2

To determine the effect of E2 on global miRNA expression in monocytic cells, miRNA profiling was carried out on THP-1 cells treated \pm E2 using TLDA. Of 768 miRNAs profiled, 25 miRNAs were identified as downregulated (relative quantification, RQ, of $<0.6$; Table 2 ) and 56 were upregulated (RQ >2.0; Table 3) in response to E2 treatment, when normalised to vehicle control (EtOH) treatment.

Given that E2 downregulates SLPI expression and that it also upregulates the expression of a range of miRNAs in THP-1 cells, the possibility that E2-induced miRNA regulation of SLPI occurs was examined. A selection of target prediction databases (TargetScanHuman 6.2, miRanda and PITA) was interrogated to identify miRNAs predicted to regulate SLPI (NM_003064.2). In total, 211 MREs were predicted in the $3^{\prime} \mathrm{UTR}$ of the SLPI mRNA. Two of the miRNAs that were identified as potentially regulating SLPI across these 3 prediction databases, hsamiR-19a and hsa-miR-19b (Fig. 2a), were upregulated by E2 treatment in THP-1 cells, as determined by miRNA profiling. Both of these miRNAs had good mirSVR scores of -1.1873 as determined by the miRanda algorithm (microRNA.org) indicating a high probability of targeting SLPI.

Next, in order to determine that this effect of E2 on was not unique to THP-1 cells, qRT-PCR assays were performed for hsa-miR-19a and hsa-miR-19b in another monocytic cell line, U937 (Fig. 2b). Basal miR-19b levels were significantly higher than miR-19a in both U937 ( $p=$ $0.0060)$. This effect was also evident in THP-1 $(p=0.0049$, data not shown). Cells were treated with either $10^{-8} \mathrm{M} \mathrm{E2}$ or EtOH. The effect of E2 on mature miR-19a and miR$19 \mathrm{~b}$ levels was examined by qRT-PCR. An increase in the expression of both miRNAs post-E2 treatment was observed in U937 cells with respect to time. This increase due to E2 treatment was statistically significant for both miRNAs after $6 \mathrm{~h}$ (miR-19a: $p=0.0124$, miR-19b: $p=$ 0.0016).

\section{miRNA Modulation Affects SLPI Expression in Monocytic Cells}

As bioinformatic analysis revealed that the miRNAs hsa-miR-19a and hsa-miR-19b are predicted to regulate SLPI, the effect of modulation of these miRNA levels on SLPI expression was investigated. Synthetic pre-miRs for miR-19a and miR-19b or scrambled non-targeting controls were transfected into U937 cells. Transfection efficiency was evaluated by analysis of fluorescent microscope images of cells transfected with fluorescent miRNA (Dharmacon Miridian miRNA-Dy547). These assessments repeatedly showed at least $70 \%$ efficiency. Fortyeight hours post-transfection, cells were lysed and RNA was isolated. qRT-PCR analysis was carried out for SLPI expression (Fig. 3). Significant knockdown (63\%, $p<$ $0.01)$ of SLPI mRNA occurred with transfection of pre- 
Table 3. miRNAs up-regulated in THP-1 monocytic cells in response to estrogen treatment

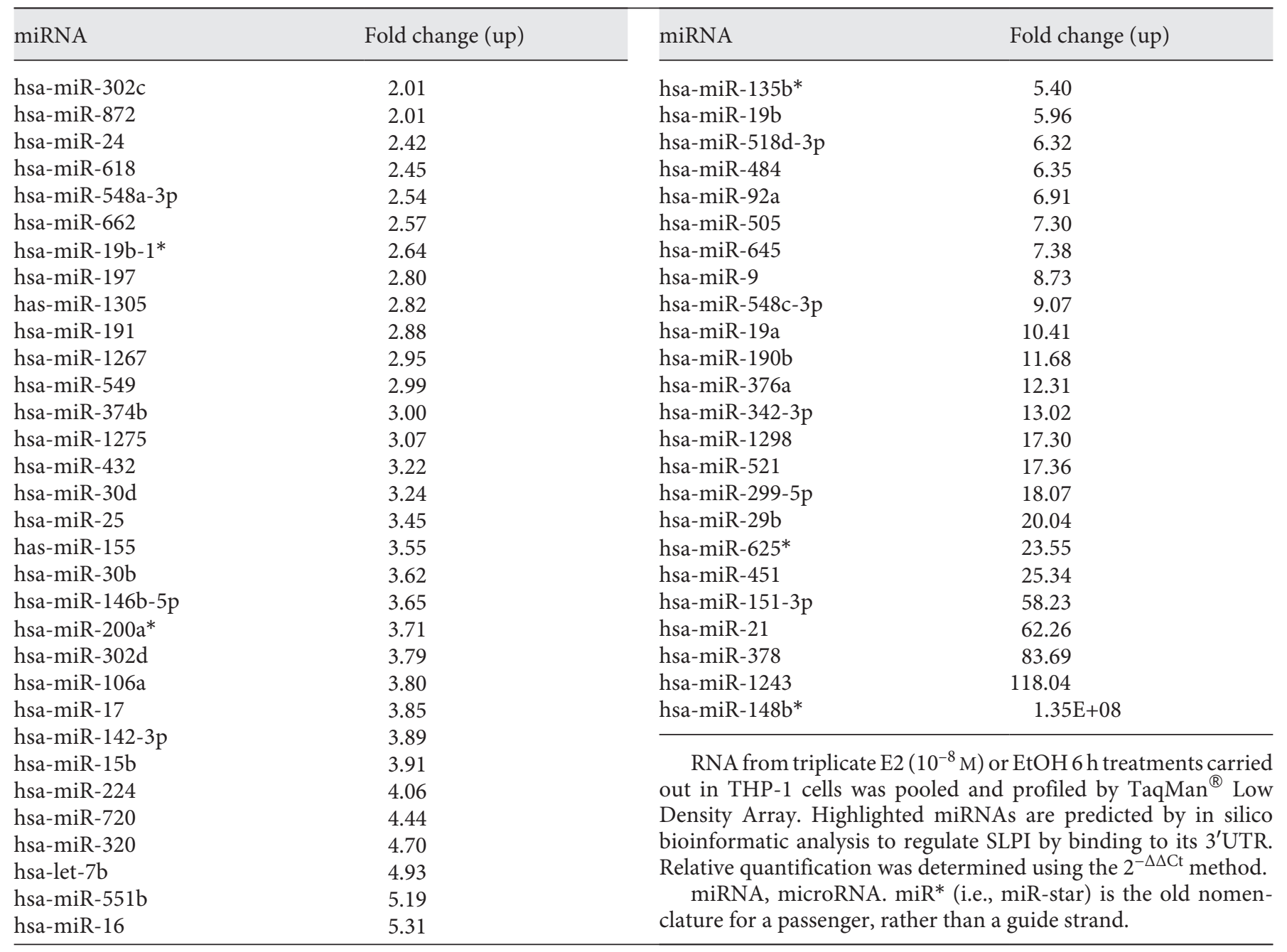

miR-19b, to a greater extent than pre-miR-19a, as compared to scrambled control (Scr; Fig. 3a). This result was mirrored at the protein level, as determined by ELISA, although protein knockdown was statistically significant using both pre-miRs (Fig. 3b).

Conversely, transfection of these cells with synthetic antimiRs for inhibition of miR-19a and miR-19b resulted in an increase in SLPI mRNA in U937 cells. This increase was only statistically significant in anti-miR-19b transfected cells, which resulted in almost 6-fold higher SLPI mRNA levels $(p=0.045)$ as compared to Scr (Fig. 3c). An effect on SLPI protein production was only seen when miR-19b levels were modulated (by antimiR$19 b)$, resulting in an increase in SLPI at the protein level (Fig. 3d).

Estrogen-Induced miR-19b Regulates SLPI in Monocytes
miR-19 Directly Binds to the SLPI 3'UTR

In order to confirm whether SLPI is a molecular target of miR-19b, pMIR-SLPI-3'UTR or pMIR-SLPI$3^{\prime}$ UTR- $\Delta 19 \mathrm{a} / \mathrm{b}$ was transiently transfected into HEK293 cells (Fig. 4) and co-transfected with either pre-miR19 a, pre-miR-19b or Scr. Co-transfection with premiR-19a or pre-miR-19b resulted in a significant decrease in luciferase gene expression from the reporter vector containing the wild-type pMIR-SLPI-3'UTR when compared with a Scr (Fig. 4a). There was no decrease in gene expression from the reporter vector containing the deleted miR-19-binding site pMIR-SLPI$3^{\prime}$ UTR- $\Delta 19 a / b$, when compared with a Scr, demonstrating direct targeting by miR-19 (miR-19a: $p<0.01$; miR-19b: $p<0.001$ ). 


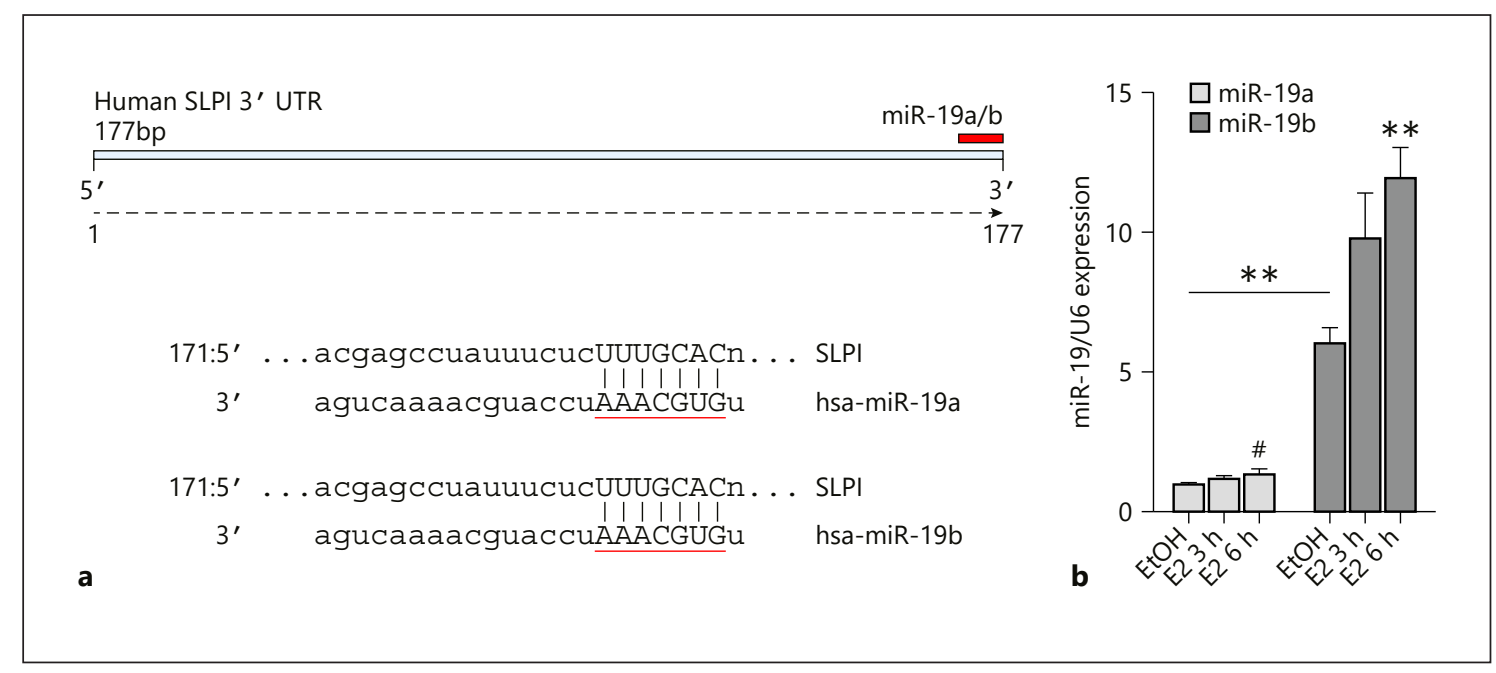

Fig. 2. miR-19a and miR-19b are increased in monocytic cells in response to E2 and are predicted to target the $3^{\prime} \mathrm{UTR}$ of SLPI. a $\mathrm{miR}-19 \mathrm{a} / \mathrm{b}$ is predicted by TargetScanHuman (version 6.2) to target a site at the distal end of the $3^{\prime}$ UTR of human SLPI. Alignment of miRNAs $19 \mathrm{a}$ and $19 \mathrm{~b}$ with their predicted target SLPI mRNA, as illustrated by TargetScanHuman (version 6.2) and microRNA.org, The 7mer-m8 (exact match to positions $2-7$ of the miRNA followed by an exact match at position 8 ) seed region is underlined. b Validation of E2-mediated induction of miR-19 in U937 cells. U937s cells $\left(1 \times 10^{5} / \mathrm{mL}\right.$, in triplicate $)$ were treated with $\mathrm{EtOH}$ or $\mathrm{E}_{2}\left(10^{-8} \mathrm{M}\right)$ for 3 or $6 \mathrm{~h}$. miR-19a and miR-19b levels were measured by qRT-PCR. Results (mean \pm SEM, $n=3$ ) are normalised to U6 snRNA expression. \# $p \leq 0.05$, ${ }^{* *} p \leq 0.01$ versus EtOH or miR-19a versus miR-19b as indicated; one-tailed Student $t$ test. SLPI, secretory leucoprotease inhibitor; EtOH, ethanol vehicle control; E2, estradiol.
Fig. 3. Modulation of miR-19a/b and SLPI in U937 cells using pre-miRNA mimics. U937 cells $\left(1 \times 10^{5} / \mathrm{mL}\right.$, in triplicate $)$ were transfected with a Scr, and either pre-miR (PM) mimics to or antimiR (AM) inhibitors against miR-19a and miR-19b for $48 \mathrm{~h}$. a, b SLPI mRNA and SLPI protein (pg/ $\mathrm{mL}$ ) in cell supernatants, respectively, post transfection with pre-miR mimics. c, d SLPI mRNA and SLPI protein in cell supernatants, respectively, post-transfection with antimiR inhibitors. mRNA and protein levels were measured by qRT-PCR (normalised to GAPDH mRNA) or ELISA, as appropriate. Results shown are representative of experiments carried out $n=3$. ${ }^{*} p \leq 0.05,{ }^{* *} p \leq 0.01$; ns vs. Scr, one-tailed $t$ test. SLPI, secretory leucoprotease inhibitor; Scr, scrambled control; ns, not significant.
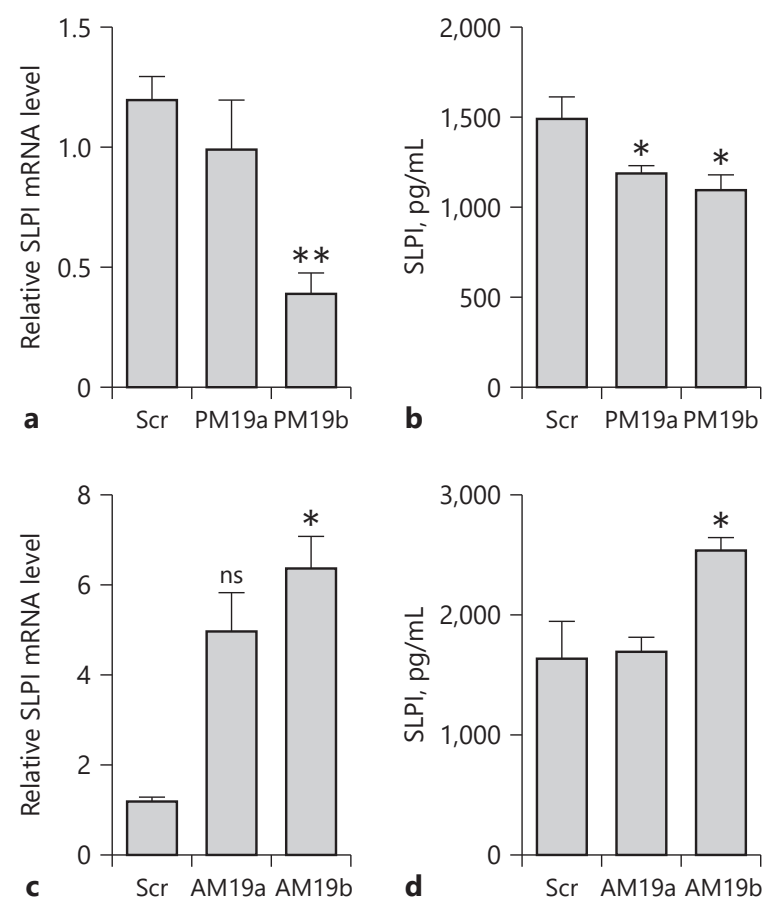


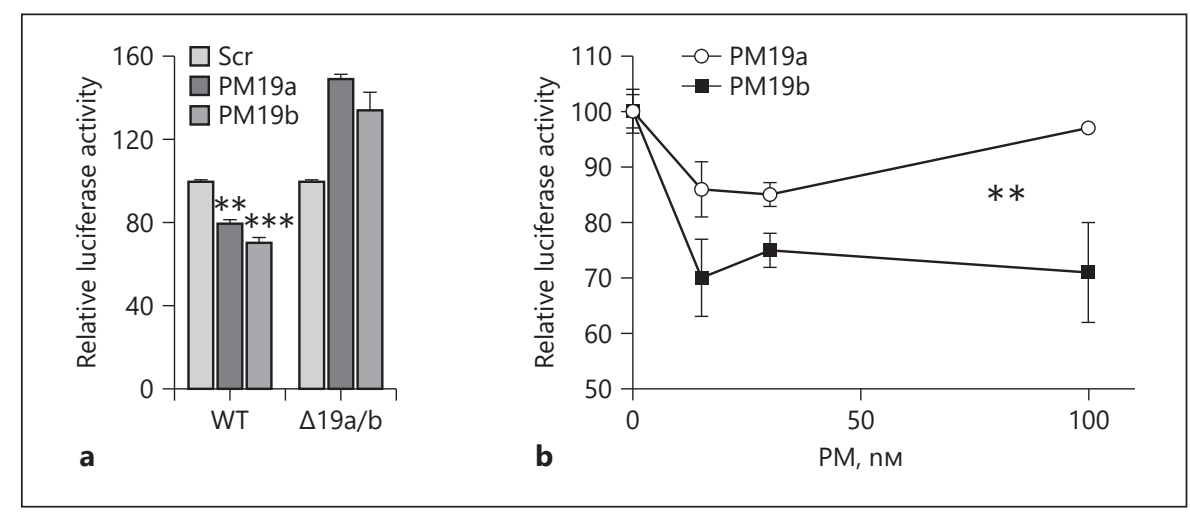

Fig. 4. miR-19 directly targets SLPI. HEK 293 cells $\left(1 \times 10^{5} / \mathrm{mL}\right.$, in triplicate) were transiently transfected with pRLSV40 (encoding Renilla luciferase, $r$-luc), pMIR-SLPI-3'UTR (WT) or pMIR-SLPI$3^{\prime}$ UTR- $\Delta 19 a / b$ (MUT; both encoding Firefly luciferase, $f$-luc), and co-transfected with either pre-miR-19a (PM19a), pre-miR-19b (PM19b) or Scr pre-miR (Scr). a The effects of pre-miR-19a and pre-miR-19b on luciferase activity $48 \mathrm{~h}$ post-transfection of
HEK293 cells with pMIR-SLPI-3'UTR or pMIR-SLPI-3'UTR$\Delta 19 a / b$. b The effects of pre-miR concentration on luciferase activity $48 \mathrm{~h}$ post-transfection of HEK293 cells co-transfected with pMIR-SLPI-3'UTR. $f$-luc activity were normalized to $r$-luc activity. Data are represented as mean \pm SEM. Data from (a) were compared by one-tailed $t$ test; ${ }^{* *} p \leq 0.01 ;{ }^{* * *} p \leq 0.001(n=3)$. Data from (b) were compared by 2 -way ANOVA; ${ }^{* *} p \leq 0.01(n \geq 2)$.

Table 4. Summary of observed effects of E2 on SLPI in monocytic cells

\begin{tabular}{llll}
\hline & THP1 & U937 & $1^{\circ}$ monocytes \\
\hline SLPI mRNA & $\downarrow$ & $\downarrow$ & $\downarrow$ \\
SLPI protein & $\downarrow$ & $\downarrow$ & nd \\
miR-19a/b & $\uparrow$ (profiling) & $\uparrow$ (miR assay) & nd \\
miR-17HG & $\uparrow$ & $\uparrow$ & nd \\
miR-17HG promoter occupancy & nd & c-MYc and ERa & nd \\
\hline
\end{tabular}

$1^{\mathrm{o}}$, primary; nd, not determined; SLPI, secretory leucoprotease inhibitor.

To distinguish the effect of miR-19a and miR-19b on SLPI expression increasing concentrations of pre-miR19a and pre-miR-19b were co-transfected with the SLPI 3'UTR reporter plasmids into HEK293 cells. Luciferase gene expression was reduced by both pre-miRs (Fig. 4b). However, pre-miR-19b had a more potent inhibitory effect than pre-miR-19a transfection at all concentrations examined $(p<0.01)$, indicating that miR-19b is a stronger regulator of SLPI than miR-19a.

\section{Estrogen-Mediated Upregulation of the miR-17-92}

Cluster Host Gene Is Mediated by c-MYC and ER $\alpha$

The miRNAs miR-19a and miR-19b-1 are encoded by the miR-17-92 cluster host gene (MIR17HG). This gene is a non-protein coding gene located on chromosome 13 and encodes a polycistronic primary transcript that yields at least 6 mature miRNAs including miR-17, miR-18,
miR-19a, miR-19b-1, miR-20 and miR-92 (Fig. 5a). The effect of E2 treatment on the expression of this transcript in monocytes was examined. THP-1 and U937 cells were treated in triplicate with either $10^{-8} \mathrm{M} \mathrm{E} 2$ or EtOH control. qRT-PCR was performed to determine mRNA levels. There was no difference for time points 3, 48 and 72 for U937 (data not shown). However, E2 significantly upregulated the expression of MIR17HG at 6- and 24-h post-E2 treatment in both cell lines examined (Fig. 5b, c).

In addition to chromosome $13, \mathrm{miR}-19 \mathrm{~b}$ is also encoded on the X chromosome (as miR-19b-2) from the paralogous miR-106a-363 cluster. The expression of this locus in response to estrogen in U937s was also examined, but this transcript was not expressed in these cells and E2 did not stimulate its expression (data not shown). Although E2 has been shown to increase the expression of the miR17-92 cluster via transactivation by the transcrip- 


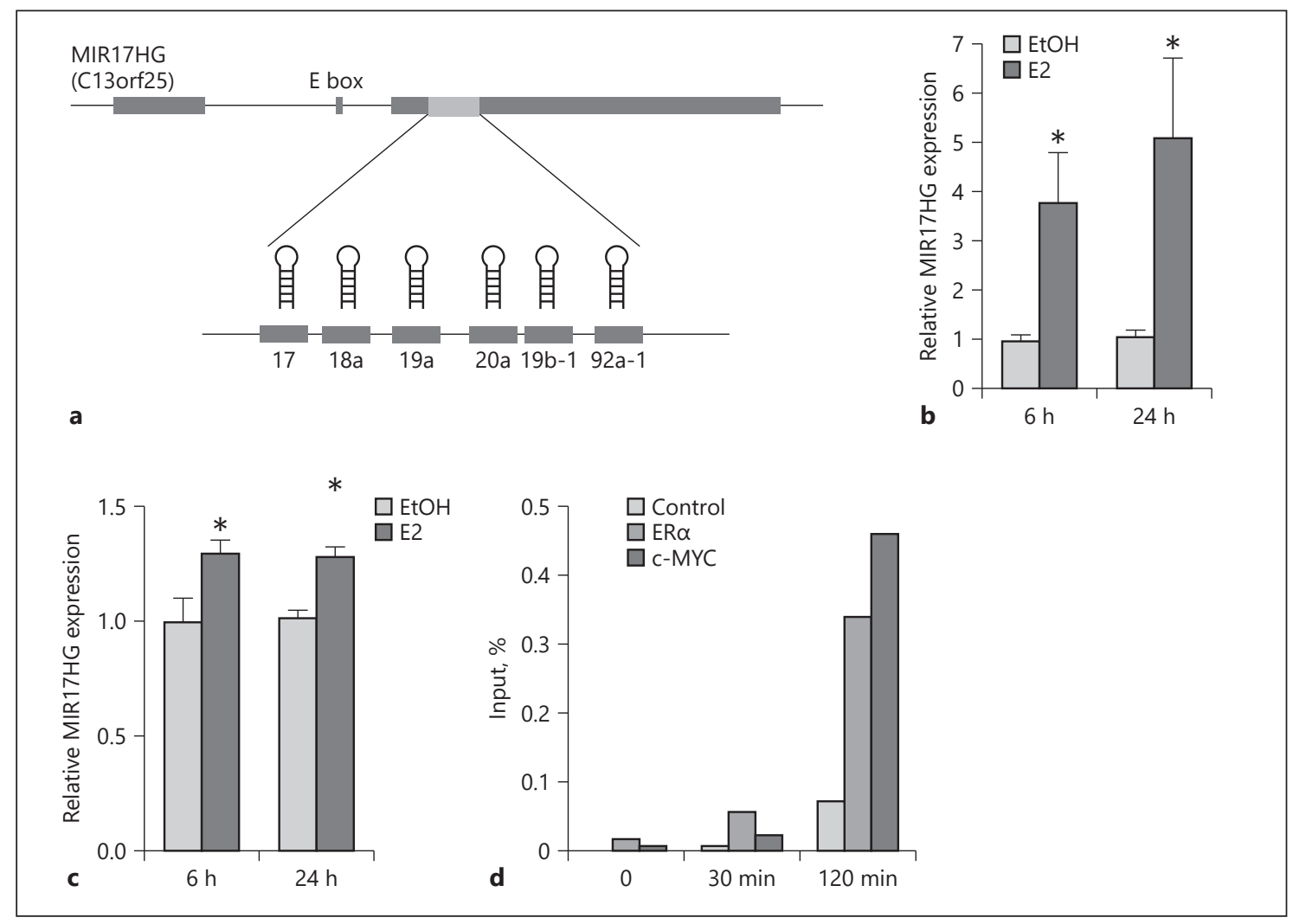

Fig. 5. MIR17HG encodes miR-19 and is controlled by ER and cMYC in response to E2 in monocytes. a Schematic of the MIR17HG transcript. MIR17HG is the host gene for the miR-17-92 cluster of 6 miRNAs, including miR-19a and miR-19b-1. Also depicted is a conserved $\mathrm{E}$ box sequence in the promoter region for this gene. THP- 1 cells $(\mathbf{b})$ or U937 cells $\left(\mathbf{c} ; 1 \times 10^{5} / \mathrm{mL}\right.$, in triplicate) were treated with vehicle control $(\mathrm{EtOH})$ or $\mathrm{E}_{2}\left(10^{-8} \mathrm{M}\right)$ for 6 or $24 \mathrm{~h}$. MIR17HG levels were measured by qRT-PCR. Results (mean \pm SEM, $n=3$ ) are normalised to GAPDH expression. THP- 1 and U937 data are representative of 2 and 3 independent experiments,

tion factor c-MYC in breast cancer cells, little is known about the E2-specific molecular interactions at this locus in monocytic cells [38]. We hypothesised that E2-mediated upregulation of miR-19 expression occurs via binding of c-MYC and ERa to a regulatory region in the MIR17HG. ChIP assays showed similar enrichment of E2-induced c-MYC and ERa DNA binding within the MIR17HG promoter (Fig. 5d).

\section{Discussion}

It is believed that sex differences in susceptibility to and progression of certain chronic inflammatory diseases are linked in part to steroid sex hormones such as estro- respectively. d U937 cells $\left(1 \times 10^{7}\right)$ were treated with $\mathrm{EtOH}$ or $\mathrm{E}_{2}$ $\left(10^{-8} \mathrm{M}\right)$ for various time points. ChIP was carried out to determine MIR17HG promoter occupancy. Cells were treated with $1 \%$ formaldehyde and subjected to ChIP analysis using c-MYC IgG, ERa IgG or non-immune IgG, as a control. The immunoprecipitated complexes were quantified by PCR using specific primers designed to amplify putative response elements. The ChIP data were normalized to input data. Data are representative of 3 independent experiments. ${ }^{*} p \leq 0.05$; one-tailed Student $t$ test. EtOH, ethanol vehicle control; E2, estradiol.

gen. Our group has previously reported that E2 increases the expression of SLPI in bronchial epithelial cells leading to a state of TLR hypo-responsiveness in these cells [5]. Here we have examined the relationship between E2 and SLPI expression in monocytes, key cells that regulate the immune response to invading pathogens (Table 4). Figure 6 summarises the mechanism underlying the findings presented here. Unexpectedly, SLPI expression was determined to be downregulated in response to $\mathrm{E} 2$ in monocytes. This was the case not only in the monocytic cell lines U937 and THP-1 but also in primary human monocytes isolated from peripheral blood.

We hypothesised that E2-induced miRNAs play a role in regulating SLPI expression in monocytes. To this end, we carried out miRNA profiling to determine the global 


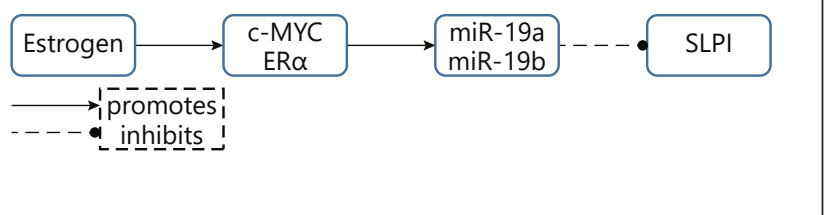

Fig. 6. Mechanism by which estrogen downregulates SLPI in monocytic cells. Estrogen promotes binding of the transcription factors C-MYC, and to a lesser extent ERa, to the promoter of the miR-17 host gene thereby leading to increased expression of miR19a and, in particular, miR-19b. Both miRNAs can bind to the single miR-19a/b site in the SLPI 3'UTR; however; miR-19b has a stronger effect than miR-19a on inhibition of SLPI expression. SLPI, secretory leucoprotease inhibitor.

miRNA expression in response to $\mathrm{E} 2$ in these cells. Of the 768 miRNAs examined, a total of 81 were differentially expressed in E2-treated monocytic cells compared to control. E2 is well-known to regulate the expression of a wide variety of miRNAs, and our results sit well with other studies which have found similar widespread E2-controlled changes in miRNA expression [32-36]. Most of these studies have been carried out in breast cancer cells, and to our knowledge, this is the first study to examine E2-regulated miRNA in monocytes.

The results outlined here confirm that E2 upregulates miR-19, as seen in other studies using the MCF-7 (human breast adenocarcinoma) cell line [32, 39]. The E2-mediated induction of MIR17HG (the pri-mir-17-92 transcript that encodes 6 miRNAs including miR19a and miR-19b-1 as part of the miR-17-92 cluster) observed here in monocytic cells has also been observed in other cells, including breast cancer cells [34, 39]. The miR-1792 cluster (also known as OncomiR-1) was originally identified as being overexpressed in B cell lymphoma due to chromosome 13q31.3 amplification [37]. miR17-92-deficient mice display neonatal lethality with developmental defects in lung hypoplasia and ventricular septal defects [40]. These and other studies suggest that this cluster has an anti-apoptotic role. miR-19, and specifically miR-19b, has also been shown to positively regulate NF- $\mathrm{kB}$ signalling, at least in primary fibroblast-like synoviocytes, by acting on repressors of NF- $\kappa B$ signalling, such as members of the A20/Tnfaip3-ubiquitin editing complex [41]. Interestingly, we determined that miR-19b expression in U937 cells is produced from this cluster on chromosome 13 and not its paralogous cluster; miR106a-363 on the X chromosome. Unfortunately, it was not possible at this time to determine whether the latter

Estrogen-Induced miR-19b Regulates

SLPI in Monocytes is expressed in primary monocytes or induced in response to E2. Regarding miR-19a, its upregulation in asthma has been shown to influence $\mathrm{T}$ cells and in particular to modulate Th2 cytokine production in the lung [42]. Furthermore, miR-19a participates in allergic lung inflammation by promoting IL-13 and IL-5 production from type 2 innate lymphoid cells [43].

The individual miRNAs encoded by the miR-17-92 polycistron have both pro- and anti-inflammatory validated targets. For example, miR-17 and miR-20a target signal-regulatory protein $\alpha$, a negative regulator of inflammation, thus increased MIR17HG potentially increases TNF- $\alpha$, IL- 6 and nitric oxide [44]. In contrast, miR-92 suppresses inflammatory responses via targeting MKK4 kinase, which would be expected to reduce TNF- $\alpha$ and IL-6 production [45]. Therefore, the overall consequence of $\mathrm{E} 2$ on the balance between the individual effects of the miR-17-92 miRNAs is likely to be complex and, as we have reported for other miRNAs, to be highly cell- and context specific [46].

Bioinformatic analysis revealed a binding site for both miR-19a and miR-19b at the end of the SLPI 3'UTR. miRNA binding sites located toward the distal end of $3^{\prime}$ UTRs have been shown to be more effective in target repression and this is potentially due to increased site accessibility [47]. Indeed both miR-19a and miR-19b were found to have good mirSVR scores, predicted by the Miranda tool [48], indicating a high probability of targeting SLPI. Both were subsequently confirmed to be upregulated by E2 in monocytic cells in additional samples and modulation of these by using pre-miRs and anti-miRs evidently modulated SLPI expression. Direct binding of both miRNAs to a predicted site at the end of the SLPI $3^{\prime} U T R$ was confirmed by luciferase assay using a full-length SLPI $3^{\prime}$ UTR inserted immediately downstream of the firefly luciferase coding sequence. A full-length $3^{\prime} U T R$ was utilised to more closely replicate the in vivo accessibility of MREs to miRNAs, including all potential RNA secondary structures and folding, that may be altered with shorter $3^{\prime}$ UTRs [49].

The results presented here demonstrate that miR-19b is more effective in targeting SLPI than miR19a. This is to a certain extent unexpected, as the sequence difference between these miRNAs is only a single nucleotide $(U>C)$ at position 11. Being 3 nucleotides away from the seed region, no difference in SLPI mRNA binding is predicted using well-established target prediction databases such as miRanda and TargetScan. In a study by Philippe et al. [50], both miRNAs have been experimentally validated to target TLR2 mRNA, with apparent equal efficiency. It is

J Innate Immun 2020;12:90-102

DOI: $10.1159 / 000500419$ 
unknown why there is such a difference in SLPI mRNA binding and knockdown between these miRNAs in monocytic cells. The single nucleotide difference may potentially result in greater affinity or more favourable binding to SLPI mRNA. It may also be explained by the basal levels of both of these miRNAs in the cells used, with miR-19b levels over 5 times higher than miR-19a levels. Although the levels of these miRs in HEK293 cells were not determined, published sequencing data suggest that the basal expression of miR-19b is also higher than miR$19 \mathrm{a}$ in these cells [51]. Interestingly, this study is not the only one to show a variance between these 2 miRNAs in function. Gantier et al. [41] have also shown that the effect of miR-19a and miR-19b is not fully redundant with miR-19b being the more effective of the 2 at suppressing negative regulators of NF- $\kappa \mathrm{B}$.

Here we also investigated the molecular mechanism by which E2 increases the expression of miR-19a and miR$19 \mathrm{~b}$ in monocytic cells. It has been previously demonstrated that some E2 responsive genes contain both cMYC and ER-binding sites located within close proximity in their promoters [52]. It has also been postulated that E2 can increase the expression of the miR 17-92 cluster via transactivation by c-MYC $[32,38]$. Indeed, Castellano et al. [32] identified a conserved ERE half site and a cMYC consensus site (E-box) in close proximity within the miR-17-92 promoter region and showed promoter occupancy by c-MYC and not ER, in response to E2, at least in breast cancer cells. In an approach to determine whether the E2-induced upregulation of miR-19 in monocytes occurs through either altered DNA methylation or increased c-MYC expression, we determined that neither the levels of the DNMT1 nor c-MYC are significantly altered by E2 treatment in monocytic cells (data not shown). However, our ChIP experiments determined that c-MYC, most likely in combination with $\mathrm{ER} \alpha$, actively binds to the miR-17-92 cluster upon E2 treatment.

The difference between the effects of E2 on SLPI expression in the lung epithelium [5] versus monocytes may be explained by the difference in basal levels of miR-19 between these cells. Basal levels of both miR-19a and -19b are significantly higher (30- to 50-fold, respectively) in monocytes compared to bronchial epithelial cells or bronchial brushings (data not shown), implying that miRNA-based regulation by miR-19 may be more significant in monocytes.

Having determined that E2 downregulates SLPI in monocytes via miRNA activity, it is unclear as to the clinical significance of this observation. E2 has been shown to regulate SLPI expression in many tissues such as mu- cosal epithelia. Most studies indicate a positive correlation between E2 and SLPI levels [30-32, 53] as opposed to the negative correlation we report in monocytes. SLPI is a multifunctional protein, with anti-protease, immunemodulatory and antimicrobial roles. It can antagonise activation of NF- $\kappa \mathrm{B}$ and inhibit subsequent pro-inflammatory cytokine production [27-29]. Thus, high E2 concentrations that reduce SLPI expression may aid in priming cells to be ready to produce pro-inflammatory mediators in response to microbial stimuli, such as LPS. This may help to explain why females generally fare better with sepsis [54-56], although studies such as these are beyond the scope of the current work and will be addressed in the future. In contrast to monocytes, which are specialised innate immune cells, the primary functions of bronchial and other epithelial cells are to act as a barrier and mediate ion transport, therefore mechanisms to rapidly modulate anti-inflammatory protein expression such as we propose here would be less important in these cells.

Limitations of this study include that pooled samples were used for miRNA profiling; however, validation of the selected miRNAs was confirmed in additional samples. It was not possible to replicate all of the cell line studies using peripheral blood monocytes or macrophages, nor was it feasible to demonstrate the effect of E2 on SLPI in the presence of miR-19b antagonism, which one would expect to be inhibited.

In summary, we have shown that $17 \beta$-E2, the primary estrogen in circulation in non-pregnant females, downregulates the expression of SLPI in human monocytes. We demonstrate a widespread change in the miRNA profile of monocytes in response to E2 and describe for the first time that the expression of SLPI is controlled through the interaction of targeting miRNA. In particular, we describe the mechanism of E2-mediated induction of miR19 in monocytes, and its ability to bind directly to the 3'UTR of SLPI, with the capacity to reduce its expression. Elsewhere we have demonstrated the effect of PLGA nanoparticles encapsulating a miR-19b mimic on SLPI expression in PMA-differentiated U937 cells [57]. This proof-of-concept study demonstrated that miR-19b-targetting nanoparticles can effectively alter target gene expression in macrophages. Given the importance of monocyte-derived macrophages in lung inflammatory processes, it will be interesting to explore the expression and function of miR-19b in alveolar macrophages in future studies and to evaluate the therapeutic potential of an anti-miR-19b approach to increase SLPI expression in those cells. 


\section{Acknowledgment}

We thank Prof. Raymond Stallings (Royal College of Surgeons in Ireland) for kindly offering use of TLDA.

\section{Disclosure Statement}

The authors have no conflicts of interest to declare.

\section{Funding Sources}

This work was funded by the Health Research Board of Ireland under Grant No. PHD/2007/11, a Vertex Innovation Award to CMG and by a European Respiratory Society Fellowship STRTF 2015 to $\mathrm{PMcK}$.

\section{References}

1 Pan Z, Chang C. Gender and the regulation of longevity: implications for autoimmunity. Autoimmun Rev. 2012 May;11(6-7):A393403.

2 Lindsay M, Howden JA. Age and Sex composition: 2010, Census Briefs. US Census Bureau; 2010.

3 Bouman A, Heineman MJ, Faas MM. Sex hormones and the immune response in humans. Hum Reprod Update. 2005 Jul-Aug;11(4): 411-23.

4 Casimir GJ, Lefèvre N, Corazza F, Duchateau J. Sex and inflammation in respiratory diseases: a clinical viewpoint. Biol Sex Differ. 2013 Sep;4(1):16.

5 Chotirmall SH, Greene CM, Oglesby IK, Thomas W, O'Neill SJ, Harvey BJ, et al. $17 \beta$-estradiol inhibits IL-8 in cystic fibrosis by up-regulating secretory leucoprotease inhibitor. Am J Respir Crit Care Med. 2010 Jul; 182(1):62-72.

6 Jackson AD, Daly L, Jackson AL, Kelleher C, Marshall BC, Quinton HB, et al. Validation and use of a parametric model for projecting cystic fibrosis survivorship beyond observed data: a birth cohort analysis. Thorax. 2011 Aug;66(8):674-9.

7 Chotirmall SH, Greene CM, Harvey BJ, McElvaney NG. The Cystic Fibrosis 'Gender Gap': Past Observations Present Understanding and Future Directions. In: Sriramulu D, editor. Cystic Fibrosis - Renewed Hopes Through Research. InTech; 2012.

8 Corey M, Farewell V. Determinants of mortality from cystic fibrosis in Canada, 19701989. Am J Epidemiol. 1996 May;143(10): 1007-17.

9 Dodge JA, Lewis PA, Stanton M, Wilsher J. Cystic fibrosis mortality and survival in the UK: 1947-2003. Eur Respir J. 2007 Mar;29(3): $522-6$.

10 FitzSimmons SC. The changing epidemiology of cystic fibrosis. J Pediatr. 1993 Jan;122(1): $1-9$.

11 Rosenfeld M, Davis R, FitzSimmons S, Pepe M, Ramsey B. Gender gap in cystic fibrosis mortality. Am J Epidemiol. 1997 May;145(9): 794-803.
12 Kulich M, Rosenfeld M, Goss CH, Wilmott R Improved survival among young patients with cystic fibrosis. J Pediatr. 2003 Jun;142(6): 631-6.

13 Block JK, Vandemheen KL, Tullis E, Fergusson D, Doucette S, Haase D, et al. Predictors of pulmonary exacerbations in patients with cystic fibrosis infected with multi-resistant bacteria. Thorax. 2006 Nov;61(11):969-74.

14 Corey M, Edwards L, Levison H, Knowles M. Longitudinal analysis of pulmonary function decline in patients with cystic fibrosis. J Pediatr. 1997 Dec;131(6):809-14.

15 Demko CA, Byard PJ, Davis PB. Gender differences in cystic fibrosis: pseudomonas aeruginosa infection. J Clin Epidemiol. 1995 Aug; 48(8):1041-9.

16 Olesen HV, Pressler T, Hjelte L, Mared L, Lindblad A, Knudsen PK, et al.; Scandinavian Cystic Fibrosis Study Consortium. Gender differences in the Scandinavian cystic fibrosis population. Pediatr Pulmonol. 2010 Oct; 45(10):959-65.

17 Levy H, Kalish LA, Cannon CL, García KC, Gerard C, Goldmann D, et al. Predictors of mucoid Pseudomonas colonization in cystic fibrosis patients. Pediatr Pulmonol. 2008 May;43(5):463-71.

18 Verma N, Bush A, Buchdahl R. Is there still a gender gap in cystic fibrosis? Chest. 2005 Oct; 128(4):2824-34.

19 Viviani L, Bossi A, Assael BM; Italian Registry for Cystic Fibrosis Collaborative Group. Absence of a gender gap in survival. An analysis of the Italian registry for cystic fibrosis in the paediatric age. J Cyst Fibros. 2011 Sep;10(5): 313-7.

20 Chotirmall SH, Smith SG, Gunaratnam C, Cosgrove S, Dimitrov BD, O'Neill SJ, et al. Effect of estrogen on pseudomonas mucoidy and exacerbations in cystic fibrosis. N Engl J Med. 2012 May;366(21):1978-86.

21 Osman M. Therapeutic implications of sex differences in asthma and atopy. Arch Dis Child. 2003 Jul;88(7):587-90.

22 Almqvist C, Worm M, Leynaert B; working group of GA2LEN WP 2.5 Gender. Impact of gender on asthma in childhood and adolescence: a GA2LEN review. Allergy. 2008 Jan; 63(1):47-57.
23 Chen W, Mempel M, Schober W, Behrendt H, Ring J. Gender difference, sex hormones, and immediate type hypersensitivity reactions. Allergy. 2008 Nov;63(11):1418-27.

24 Kynyk JA, Mastronarde JG, McCallister JW. Asthma, the sex difference. Curr Opin Pulm Med. 2011 Jan;17(1):6-11.

25 Han MK, Postma D, Mannino DM, Giardino ND, Buist S, Curtis JL, et al. Gender and chronic obstructive pulmonary disease: why it matters. Am J Respir Crit Care Med. 2007 Dec;176(12):1179-84.

26 Machado MC, Krishnan JA, Buist SA, Bilderback AL, Fazolo GP, Santarosa MG, et al. Sex differences in survival of oxygen-dependent patients with chronic obstructive pulmonary disease. Am J Respir Crit Care Med. 2006 Sep; 174(5):524-9.

27 Jin FY, Nathan C, Radzioch D, Ding A. Secretory leukocyte protease inhibitor: a macrophage product induced by and antagonistic to bacterial lipopolysaccharide. Cell. 1997 Feb; 88(3):417-26.

28 Ding A, Thieblemont N, Zhu J, Jin F, Zhang J, Wright S. Secretory leukocyte protease inhibitor interferes with uptake of lipopolysaccharide by macrophages. Infect Immun. 1999 Sep;67(9):4485-9.

29 Taggart CC, Cryan SA, Weldon S, Gibbons A, Greene CM, Kelly E, et al. Secretory leucoprotease inhibitor binds to NF-kappaB binding sites in monocytes and inhibits p65 binding. $J$ Exp Med. 2005 Dec;202(12):1659-68.

30 Chen D, Xu X, Cheon YP, Bagchi MK, Bagchi IC. Estrogen induces expression of secretory leukocyte protease inhibitor in rat uterus. Biol Reprod. 2004 Aug;71(2):508-14.

31 Fahey JV, Wright JA, Shen L, Smith JM, Ghosh M, Rossoll RM, et al. Estradiol selectively regulates innate immune function by polarized human uterine epithelial cells in culture. Mucosal Immunol. 2008 Jul;1(4): 317-25.

32 Castellano L, Giamas G, Jacob J, Coombes RC, Lucchesi W, Thiruchelvam P, et al. The estrogen receptor-alpha-induced microRNA signature regulates itself and its transcriptional response. Proc Natl Acad Sci USA. 2009 Sep;106(37):15732-7. 
33 Ferraro L, Ravo M, Nassa G, Tarallo R, De Filippo MR, Giurato G, et al. Effects of oestrogen on microRNA expression in hormoneresponsive breast cancer cells. Horm Cancer. 2012 Jun;3(3):65-78.

34 Masuda M, Miki Y, Hata S, Takagi K, Sakurai $\mathrm{M}$, Ono K, et al. An induction of microRNA, miR-7 through estrogen treatment in breast carcinoma. J Transl Med. 2012 Sep;10(Suppl 1):S2.

35 Saumet A, Vetter G, Bouttier M, Antoine E, Roubert C, Orsetti B, et al. Estrogen and retinoic acid antagonistically regulate several microRNA genes to control aerobic glycolysis in breast cancer cells. Mol Biosyst. 2012 Oct; 8(12):3242-53.

36 Munagala R, Aqil F, Vadhanam MV, Gupta RC. MicroRNA 'signature' during estrogenmediated mammary carcinogenesis and its reversal by ellagic acid intervention. Cancer Lett. 2013 Oct;339(2):175-84.

37 He L, Thomson JM, Hemann MT, HernandoMonge E, Mu D, Goodson S, et al. A microRNA polycistron as a potential human oncogene. Nature. 2005 Jun;435(7043):828-33.

38 O’Donnell KA, Wentzel EA, Zeller KI, Dang CV, Mendell JT. c-Myc-regulated microRNAs modulate E2F1 expression. Nature. 2005 Jun;435(7043):839-43.

39 Cochrane DR, Cittelly DM, Howe EN, Spoelstra NS, McKinsey EL, LaPara K, et al. MicroRNAs link estrogen receptor alpha status and Dicer levels in breast cancer. Horm Cancer. 2010 Dec;1(6):306-19.

40 Ventura A, Young AG, Winslow MM, Lintault L, Meissner A, Erkeland SJ, et al. Targeted deletion reveals essential and overlapping functions of the miR-17 through 92 family of miRNA clusters. Cell. 2008 Mar;132(5): 875-86.

41 Gantier MP, Stunden HJ, McCoy CE, Behlke MA, Wang D, Kaparakis-Liaskos M, et al. A miR-19 regulon that controls NF- $\kappa B$ signaling. Nucleic Acids Res. 2012 Sep;40(16): 8048-58.
42 Simpson LJ, Patel S, Bhakta NR, Choy DF, Brightbill HD, Ren X, et al. A microRNA upregulated in asthma airway $\mathrm{T}$ cells promotes TH2 cytokine production. Nat Immunol. 2014 Dec;15(12):1162-70.

43 Singh PB, Pua HH, Happ HC, Schneider C, von Moltke J, Locksley RM, et al. MicroRNA regulation of type 2 innate lymphoid cell homeostasis and function in allergic inflammation. J Exp Med. 2017 Dec;214(12):3627-43.

44 Zhu D, Pan C, Li L, Bian Z, Lv Z, Shi L, et al. MicroRNA-17/20a/106a modulate macrophage inflammatory responses through targeting signal-regulatory protein $\alpha$. J Allergy Clin Immunol. 2013 Aug;132(2):426-36.e8.

45 Lai L, Song Y, Liu Y, Chen Q, Han Q, Chen W, et al. MicroRNA-92a negatively regulates Toll-like receptor (TLR)-triggered inflammatory response in macrophages by targeting MKK4 kinase. J Biol Chem. 2013 Mar; 288(11):7956-67.

46 Hassan T, Smith SG, Gaughan K, Oglesby IK, O’Neill S, McElvaney NG, et al. Isolation and identification of cell-specific microRNAs targeting a messenger RNA using a biotinylated antisense oligonucleotide capture affinity technique. Nucleic Acids Res. 2013 Apr;41(6):e71.

47 Grimson A, Farh KK, Johnston WK, GarrettEngele P, Lim LP, Bartel DP. MicroRNA targeting specificity in mammals: determinants beyond seed pairing. Mol Cell. 2007 Jul;27(1): 91-105.

48 Betel D, Koppal A, Agius P, Sander C, Leslie C. Comprehensive modeling of microRNA targets predicts functional non-conserved and non-canonical sites. Genome Biol. 2010; 11(8):R90.

49 Kuhn DE, Martin MM, Feldman DS, Terry AV Jr, Nuovo GJ, Elton TS. Experimental validation of miRNA targets. Methods. 2008 Jan; 44(1):47-54.
50 Philippe L, Alsaleh G, Suffert G, Meyer A, Georgel P, Sibilia J, et al. TLR2 expression is regulated by microRNA miR-19 in rheumatoid fibroblast-like synoviocytes. J Immunol. 2012 Jan; 188(1):454-61.

51 Landgraf P, Rusu M, Sheridan R, Sewer A, Iovino N, Aravin A, et al. A mammalian microRNA expression atlas based on small RNA library sequencing. Cell. 2007 Jun;129(7): 1401-14.

52 Cheng AS, Jin VX, Fan M, Smith LT, Liyanarachchi S, Yan PS, et al. Combinatorial analysis of transcription factor partners reveals recruitment of c-MYC to estrogen receptor- $\alpha$ responsive promoters. Mol Cell. 2006 Feb;21(3):393-404

53 Shimoya K, Zhang Q, Temma K, Kimura T, Tsujie T, Tsutsui T, et al. Secretory leukocyte protease inhibitor levels in cervicovaginal secretion of elderly women. Maturitas. 2006 May;54(2):141-8.

54 Offner PJ, Moore EE, Biffl WL. Male gender is a risk factor for major infections after surgery. Arch Surg. 1999 Sep;134(9):935-8; discussion 938-40.

55 Diodato MD, Knöferl MW, Schwacha MG, Bland KI, Chaudry IH. Gender differences in the inflammatory response and survival following haemorrhage and subsequent sepsis. Cytokine. 2001 May;14(3):162-9.

56 Zellweger R, Wichmann MW, Ayala A, Stein $\mathrm{S}$, DeMaso CM, Chaudry IH. Females in proestrus state maintain splenic immune functions and tolerate sepsis better than males. Crit Care Med. 1997 Jan;25(1):106-10.

57 McKiernan PJ, Lynch P, Ramsey JM, Cryan SA, Greene CM. Knockdown of Gene Expression in Macrophages by microRNA MimicContaining Poly (Lactic-co-glycolic Acid) Microparticles. Medicines (Basel). 2018 Dec; 5(4):E133. 